\title{
Pigmented border of the toe as a consistent landmark to the digital nerve
}

\author{
Maddumaarachchi HSTM ${ }^{1}$, Mathangasinghe $\mathrm{Y}^{1}$, Samaranayake UMJE${ }^{1}$, Malalasekera \\ $\mathrm{Ap}^{1}$, Anthony $\mathrm{DJ}^{1}$ \\ ${ }^{\prime}$ Department of Anatomy, Faculty of Medicine, University of Colombo, Colombo, Sri Lanka
}

\section{Introduction}

The purpose of this study was to identify surface landmarks of digital nerves corresponding to the pigmented border of the toes.

\section{Methods}

A total of 140 digital nerves in 10 preserved adult cadaveric toes were studied. Photographs of the cross sections of the frozen toes at the proximal crease we analyzed using Image J (version 1.48) software. Circumferential distance to each digital nerve from the pigmented border, the direct distance to the nerve from the pigment border, the depth from the skin and the angle to the nerve from a perpendicular line at the pigmented border were measured.

\section{Results}

Majority $(95.7 \%, n=134)$ of the digital nerves were located towards the plantar aspect of the pigmented border. The median circumferential distance to the digital nerve from the pigmented border was $0.28(\mathrm{IQR}=0.14-0.42) \mathrm{mm}$. The digital nerve was located 0.29 $(\mathrm{IQR}=0.20-0.37) \mathrm{mm}$ deep to the skin in all the toes except in the big toe. In the big toe, this distance was 0.35 (IQR $=0.29$ $0.42) \mathrm{mm}$. The median angle was 33.130 $(\mathrm{IQR}=14.670-50.500)$. Kruskal-Wallis test did not show statistically significant difference in the circumferential distances $[\chi 2(4)=7.348, p=0.119]$ between toes, but there were significant differences in the direct distance from the pigment border $[\chi 2(4)=10.240, p=0.037]$ and the depth from the skin surface $[\chi 2(4)=11.897, p=0.018]$ among the toes. The post-hoc Mann-Whitney tests showed that these dimensions mainly differed in the big toe compared to the rest of the toes. Independent-samples Mann-Whitney $U$ and Kruskal-Wallis tests showed that none of these measurements significantly differed according to the gender or the side of the toe respectively $(\mathrm{p}>.05)$. Spearman's correlation coefficient found a statistically significant negative correlation between the mid-thigh circumference and the circumferential distance from the pigmented border to the nerve $(r=.225, \mathrm{p}=.008)$.

\section{Discussion and Conclusions}

The pigmented border of toe is a reliable anatomical landmark to locate digital nerves. 